\title{
The Ventral Striatum, Hypersociability, and Sleepwalking at Year's End
}

\author{
By Eric Hollander, MD
}

This month's CNS Spectrums introduces the exciting new clinical column series "Brain Regions of Interest," which is overseen by Michael Trimble, MD, FRCP, FRPsych. This column will include articles on the neuroanatomy of key brain structures important for neuropsychiatry, functional brain circuitry, and the clinical neurosciences of psychiatry and neurology. I can think of no region that is of greater topical interest than the ventral striatum, including the nucleus accumbens, which serves as an interface not only between the limbic and motor systems but also between psychiatry and neurology. It plays a unique role in the emotional and motivational aspects of behavior as well as clinical entities such as substance and behavioral addictions, obsessive-compulsive disorder, and schizophrenia.

In this month's contribution, Henk J. Groenewegen, MD, PhD, helps tease apart the role of the dorsal versus ventral regions of the striatum as well as the shell versus the core of the nucleus accumbens. In so doing, this helps explain the input and output pathways of the basal ganglia and their unique behavioral and motor effects. This has important clinical implications for interventional treatments, such as deep brain stimulation, and clinical phenomena, such as the development of impulsive and compulsive behaviors in patients with Parkinson's disease.

This issue also includes a fascinating article by Stephen I. Deutsch, MD, PhD, and colleagues on Williams syndrome, a neurodevelopmental disorder that results from the deletion of 25-30 genes. Patients with this syndrome present with the combination of a distinctive elfin-like facial appearance; growth retardation; mild mental retardation; an inconsistent cognitive profile, which includes visuospatial impairments with good facial dis- crimination and relatively preserved expressive language skills; and cardiovascular abnormalities. In addition, a striking behavioral feature of the syndrome is the high sociability and empathy that these patients show for others.

Williams syndrome has enormous heuristic value because its pathological feature of heightened "sociability" can be a "deficit" symptom of major complex neuropsychiatric disorders, such as schizophrenia and autism. From a research perspective, the syndrome lends itself to neurobiological studies of sociability as a dimension that varies independently of cognition (or at least many separable cognitive processes). From a clinical perspective, the syndrome challenges us to administer strategic psychosocial interventions that take advantage of the opportunities that pathological sociability provide, while avoiding its threats.

Somnambulism, a previously unreported side effect of quetiapine, is described in two cases by Zeba Hasan Hafeez, MD, and Constance M. Kalinowski, MD. Both cases cited here involved individuals who had no prior or family history of somnambulism and had attention-deficit/hyperactivity disorder. Somnambulism is a common parasomnia that reflects an impairment in the normal mechanisms of arousal from sleep in which motor behaviors are activated without full consciousness. Motor behaviors are initiated during deep nonrapid eye movement or slow-wave sleep (stages 3-4), and may be limited to relatively simple manifestations, such as sitting up, fumbling with objects or bedclothes, or mumbling. Comorbid anxiety disorders are frequently encountered in psychoses and mainly assessed during the hospitalization.

Antonio Ciapparelli, MD, and colleagues investigated 98 patients with schizophrenia, schizoaffective, or bipolar disorder, previously hospitalized

Dr. Hollander is the editor of this journal, Esther and Joseph Klingenstein Professor and Chairman of Psychiatry at the Mount Sinai School of Medicine, and director of the Seaver and New York Autism Center of Excellence in New York City. 
for psychotic symptoms. Assessments, including Structured Clinical Interview for the Diagnostic and Statistical Manual of Mental Disorders, Fourth Edition, Brief Psychiatric Rating Scale, and Clinical Global Impression Scale, were performed during hospitalization and in remission.

John P. O'Reardon, MD, and colleagues report a coincidental reduction in headache pain in patients treated with repetitive transcranial magnetic stimulation (rTMS) for major depressive disorder (MDD). Two patients with a DSM-IV diagnosis of MDD, non-responsive to prior antidepressant treatment who were enrolled in a sham-controlled, doubleblind study of rTMS for MDD; after the study it was revealed that both were in the active-treatment arm. Both patients suffered from nearly daily headaches and kept records of headache frequency and severity before, during, and after the study. Headache pain was significantly reduced under double-blind conditions with rTMS treatment, but returned to baseline following cessation of rTMS treatment. Ultimately, when receiving rTMS poststudy as a maintenance intervention for MDD, the positive effects on headache reduction were sustained. Headache pain is frequently comorbid with mood disorders and has been reported as the most common side effect with rTMS. In these subjects, rTMS was, in fact, associated with relief of depressive symptoms and preexisting headache pain. This indicates that rTMS may be beneficial for both disorders in some patients.
Finally, this month's "Communique" describes a patient with treatment-resistant posttraumatic stress disorder who responded to sodium oxybate, a $\gamma$-aminobutyric acid $B$ agonist. Clearly, this is an anecdotal observation and off-label use of this medication, and clinicians need to use their own clinical judgement and weigh the individual risks versus the benefits for their patients. Perhaps this observation will lead to more systematic controlled trials in this population, which has a real need for additional effective treatments.

Happy Holidays. CNS

\section{ERRATA}

In the PubMed/MEDLINE citation Marazziti D, Dell'Osso B, Dell'Osso MC, etal. Romantic Attachment in Patients with Mood and Anxiety Disorders. CNS Spectr. 2007;12(10):751-756 by-line, Dell'Osso MC should read Catena Dell'Osso $M$.

In Afzal KI, Briones DF, DeVargas C. RisperidoneInduced Polydipsia and Polyphagia Associated with Galactorrhea, Abdominal Pain, and Papid Weight Gain in an Adolescent Hispanic Female. CNS Spectr. 2007; 12(11):818-820, Dr. DeVargas is listed as assistant professor in the Department of Neuropsychiatry and chief of the Department of Child and Adolescent Psychiatry. She should be listed as assistant professor in the Department of Neuropsychiatry and chief of Child and Adolescent Psychiatry.

\title{
Now Available Online at www.cnsspectrums.com
} CME-ACCREDITED SUPPLEMENT

\author{
An expert review of clinical challenges in psychiatry
}

\section{The Management of Schizophrenia:}

\section{Safety, Treatment, and Continuity of Care}

\author{
by John M. Kane, MD, Kimberly Nordstrom, MD, JD, Michael H. Allen, MD, \\ Christoph U. Correll, MD, and Robert E. Drake, MD, PhD
}

To request a published supplement, pleasee-mail ks@mblcommunications.com

Supported by an educational grant from Janssen, L.P., administered by Ortho-McNeil Janssen Scientific Affairs, LLC. 